IFT-UAM/CSIC-09-53

DESY 09-186

LTH 852

\title{
The epsilon regime of chiral perturbation theory with Wilson-type fermions
}

\author{
K. Jansen, \\ NIC, DESY Zeuthen, Platanenallee 6 \\ D-15738 Zeuthen, Germany \\ E-mail: karl.jansen@desy.de
}

\section{A. Shindler ${ }^{* \dagger}$}

Theoretical Physics Division,

Dept. of Mathematical Sciences,

University of Liverpool

Liverpool L69 7ZL, UK

E-mail: shindlereliv.ac.uk

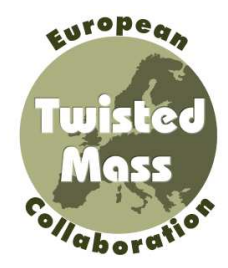

In this proceeding contribution we report on the ongoing effort to simulate Wilson-type fermions in the so called epsilon regime of chiral perturbation theory $(\chi \mathrm{PT})$. We present results for the chiral condensate and the pseudoscalar decay constant obtained with Wilson twisted mass fermions employing two lattice spacings, two different physical volumes and several quark masses. With this set of simulations we make a first attempt to estimate the systematic uncertainties.

The XXVII International Symposium on Lattice Field Theory

July 26-31, 2009

Peking University, Beijing, China

\footnotetext{
* Speaker.

†Current address:Instituto de Física Teórica UAM/CSIC Universidad Autónoma de Madrid, Cantoblanco E-28049 Madrid, Spain
} 


\section{Introduction}

The low energy dynamics of QCD can be quantitatively understood computing the universal low energy constants (LECs) of the chiral effective theory [1]. QCD results obtained with simulations on a space-time lattice can be matched with the effective theory, if the range of quark masses and energy scales in the QCD computations, are such that the higher order corrections in the effective theory calculations are well under control. A particular attractive framework in this respect is the so-called $\varepsilon$-regime [2]. In this regime the power counting for masses, momenta and linear size of the volume, is such that higher order corrections are suppressed in comparison with the standard $p$-regime. Thus this regime provides an alternative, but also complementary, way to determine the LECs in comparison with standard p-regime matchings. The $\varepsilon$-regime is properly matched if the volume in the QCD simulations, and correspondingly in the chiral perturbation theory $(\chi \mathrm{PT})$ computations, is larger than the confinement radius and if the Goldstone boson correlation length of the system is smaller than the inverse linear size of the volume. Typically the usage of GinspargWilson [3] (GW) fermions is preferred because GW fermions have a natural definition for the topological charge, thus they allow to study the $\varepsilon$-regime in a fixed topological sector [4] for which also $\chi$ PT formulae have been worked out [5].

Wilson-type fermions can still, in principle, probe the $\varepsilon$-regime, sampling all topological sectors $[6,7]$. An important point is the interplay between low quark masses and a finite lattice spacing. It can be addressed using the concept of generalized chiral expansions applied to the $\varepsilon$ regime $[8,9]$. A second issue is the algorithm which ought to be used to practically simulate in the $\varepsilon$-regime with Wilson-type fermions. In these proceedings we cover these two topics, and present preliminary results for the chiral condensate ${ }^{1} \Sigma$ and the pseudoscalar decay constant $F$. Additionally we discuss the systematic uncertainties which can affect the determination of these LECs in the $\varepsilon$-regime.

\section{2. $\varepsilon$ expansion with Wilson fermions}

The $\varepsilon$-regime has been introduced to cure the appearance of infrared divergences in $\chi \mathrm{PT}$ when the mass squared of the Goldstone boson $\left(M_{\pi}\right)$ becomes smaller than the inverse size of the box $(V=$ $\left.L^{3} \times T\right)$. To cure this problem Gasser and Leutwyler [2] proposed to change the power counting (from $p$-regime to $\varepsilon$-regime) in this particular region of the parameter space to achieve an exact resummation of the zero modes of the Goldstone bosons and removing in this way any infrared divergence. As a result of this resummation the order parameter of the chiral phase transition, the chiral condensate, vanishes in the chiral limit. This signals the expected recovery of chiral symmetry when the quark mass $m_{\mathrm{q}}$ vanishes in a finite volume, i.e. the absence of spontaneous chiral symmetry. When studying the cutoff effects with Wilson-type fermions in the framework of $\chi \mathrm{PT}$ the power counting of the $\varepsilon$-regime in the continuum

$$
\frac{1}{T}=\mathrm{O}(\varepsilon), \quad \frac{1}{L}=\mathrm{O}(\varepsilon), \quad M_{\pi}^{2}=\mathrm{O}\left(\varepsilon^{4}\right) \quad\left[\text { or } \quad m_{\mathrm{q}}=\mathrm{O}\left(\varepsilon^{4}\right)\right],
$$

has to be augmented to include the lattice spacing $a$. This is usually done connecting the power counting of the quark mass $m_{\mathrm{q}}$ with the lattice spacing times the appropriate powers of the QCD scale $\Lambda$ to restore the proper dimensions. Typically in the $p$-regime there are two different power countings depending on how the quark mass and the lattice spacing are related: the GSM regime [10] 
and the Aoki [11] or large cutoff effects (LCE) regime. In the so-called GSM regime $m_{\mathrm{q}} \sim a \Lambda^{2}$ which implies $a=O\left(\varepsilon^{4}\right)$. In this regime the leading order (LO) cutoff effects can be reabsorbed in the definition of the quark mass, implying no cutoff effects up to NLO order corrections (this is indeed true also in the $p$-regime). At NLO the chiral Lagrangian describing the dynamics of the Goldstone field $U(x)$ is given by

$$
\begin{aligned}
\mathscr{L}_{W \chi}^{(4)} & =\mathscr{L}_{\chi}^{(4)}+a \widetilde{W} \operatorname{Tr}\left(\partial_{\mu} U^{\dagger} \partial_{\mu} U\right) \operatorname{Tr}\left(U+U^{\dagger}\right)-2 a B_{0} W \operatorname{Tr}\left(\mathscr{M}^{\prime \dagger} U+U^{\dagger} \mathscr{M}^{\prime}\right) \operatorname{Tr}\left(U+U^{\dagger}\right)+ \\
& -a^{2} W^{\prime}\left[\operatorname{Tr}\left(U+U^{\dagger}\right)\right]^{2}-2 a B_{0} H^{\prime} \operatorname{Tr}\left(\mathscr{M}^{\prime}+\mathscr{M}^{\dagger \dagger}\right),
\end{aligned}
$$

where $\mathscr{L}_{W \chi}^{(4)}$ is the continuum NLO chiral Lagrangian, $W, \widetilde{W}$ and $W^{\prime}$ are LECs parametrizing cutoff effects, and $\mathscr{M}^{\prime}$ is the shifted mass matrix which reabsorbes the LO $\mathrm{O}(a)$ cutoff effects. Given our particular choice of the power counting it is easy to see that all the corrections terms to the continuum Lagrangian are of NNLO [8, 9]. This implies that Wilson fermions are "effectively" free from discretization errors up to NNLO corrections. This result is indipendent whether we use a clover term in the fermion action or not and whether we use twisted mass fermions or not.

The Aoki regime is defined by $m_{\mathrm{q}} \sim a^{2} \Lambda^{3}$ which implies $a=O\left(\varepsilon^{2}\right)$. The LO chiral Lagrangian in the Aoki regime is

$$
\mathscr{L}_{W \chi}^{(2)}=\frac{F^{2}}{4} \operatorname{Tr}\left[\partial_{\mu} U^{\dagger} \partial_{\mu} U\right]-\frac{\Sigma}{2} \operatorname{Tr}\left[\mathscr{M}^{\dagger \dagger} U+\mathscr{M}^{\prime} U^{\dagger}\right]-a^{2} W^{\prime}\left[\operatorname{Tr}\left(U+U^{\dagger}\right)\right]^{2} .
$$

It contains already at $\mathrm{LO} \mathrm{O}\left(a^{2}\right)$ cutoff effects that cannot be reabsorbed in the definition of the quark mass. It is well known $[12,13]$ that these LO cutoff effect in infinite volume change the vacuum structure of the effective theory leading to two possible scenarios for the chiral phase diagram [14]. In the Aoki scenario the pattern of spontaneous symmetry breaking changes the continuum $S U(2)_{L} \times S U(2)_{R} \rightarrow S U(2)_{V}$ into $S U(2)_{V} \rightarrow U(1)$ signalling the spontaneous breaking of flavour (and parity) symmetry. In the Sharpe-Singleton scenario there is no phase transition in the chiral limit and the Goldstone bosons remain massive. It is quite clear that in both scenarios the physics of the zero-modes is quite different from the one of the continuum or of the GSM regime. More work is needed in order to understand Wilson-type fermions in the deep chiral regime.

The $\varepsilon$-regime gives us the possibility to have a transition region between the two regime in which the cutoff effects appear at NLO $[8,9]$. To understand this regime from a power counting point of view we can decide to set $a=O\left(\varepsilon^{3}\right)$. The LO action contains, as in the continuum, the mass term $\left[S_{2}^{(0)}\right]_{\mathscr{M}}$ and the kinetic term for the non-zero-modes. First corrections due to a finite lattice spacing appear at NLO. The partition function of the effective theory at NLO can be written as $^{2}$

$$
\begin{aligned}
& \mathscr{Z}=\mathscr{N} \int \mathscr{D}\left[U_{0}\right] \mathrm{e}^{-\left[S_{2}^{(0)}\right]} \mathscr{M}^{\left(\Sigma_{\text {eff }}\right)} \times Z_{\pi}\left[U_{0}\right], \quad\left[S_{2}^{(0)}\right] \mathscr{M}=-\frac{\Sigma}{2} \int d^{4} x \operatorname{Tr}\left[\mathscr{M}^{\dagger \dagger} U_{0}+U_{0}^{\dagger} \mathscr{M}^{\prime}\right], \\
& Z_{\pi}\left[U_{0}\right]=\mathscr{N}\left\{1+W^{\prime} a^{2} V\left[\operatorname{Tr}\left(U_{0}+U_{0}^{\dagger}\right)\right]^{2}+\frac{2 a W \Sigma V}{F^{2}} \operatorname{Tr}\left[\mathscr{M}^{\prime \dagger} U_{0}+U_{0}^{\dagger} \mathscr{M}^{\prime}\right] \operatorname{Tr}\left[U_{0}+U_{0}^{\dagger}\right]\right\} .
\end{aligned}
$$

It is clear from eqs. (2.3) (2.4) that the discretization errors of $\mathrm{O}\left(a^{2}\right)$ are of NLO while the discretization errors of $\mathrm{O}\left(a m_{\mathrm{q}}\right)$ are of higher order being of $\mathrm{O}\left(\varepsilon^{3}\right)$. With this partition function it

\footnotetext{
${ }^{2} \Sigma_{\text {eff }}$ contains the NLO correction to $\Sigma$ coming from the one loop non-zero modes contribution.
} 
is straightforward to introduce appropriate sources and compute two-point functions. We refer to $[8,9]$ for details on the computation. It turns out that the relative $\mathrm{O}\left(a^{2}\right)$ cutoff effects corrections, over a wide range of values for $m_{\mathrm{q}} \Sigma V$, are at most of few percent. Another result of our analysis $[8,9]$ is that there are appropriate linear combinations of correlation functions like $C_{\mathrm{S}}\left(x_{0}\right) / 4+3 C_{\mathrm{P}}\left(x_{0}\right)$ or $C_{\mathrm{AA}}\left(x_{0}\right)+C_{\mathrm{VV}}\left(x_{0}\right)$ which are free from $\mathrm{O}\left(a^{2}\right)$ effects and have leading cutoff effects of $\mathrm{O}\left(a m_{\mathrm{q}}\right)$, i.e. no discretization errors up to NNLO. We can summarize the results of our analysis generalizing the $\varepsilon$ expansion using Wilson fermions in the following way. In the Aoki regime there could be large cutoff effects and more work is needed to completely understand the interplay between quark mass and lattice spacing effects. In the GSM regime Wilson fermions have no cutoff effects up to NNLO. In the transition region between the two regimes we have a tool to analyze cutoff effects and we have computed correlators including $\mathrm{O}\left(a^{2}\right)$ and $\mathrm{O}\left(a m_{\mathrm{q}}\right)$. The proper power counting has been identified as $a=O\left(\varepsilon^{3}\right)$ and two-point functions have been computed up to relative $\mathrm{O}\left(\varepsilon^{3}\right)$ corrections. This analytical effort obviously has to be combined with numerical simulations which can tell us which regime has been properly matched. We are currently extending this computation for Wilson-twisted mass fermions [15].

\section{Numerical results with Wilson twisted mass fermions}

To perform simulations in the $\varepsilon$-regime with Wilson-type fermions we need specific algorithmic improvements. The main ingredient for these improvements is reweighting. We first proposed to use the PHMC algorithm [16] combined with exact reweighiting to include in an exact way the low modes of the Wilson twisted mass operator [6]. Different but somehow related techinques have been later proposed based on stochastic reweighting in the standard mass [17] or in the twisted mass [18]. The main goal of reweighting in the $\varepsilon$-regime is to ensure a better sampling of the configuration space and to avoid instabilities issues with HMC-like algorithms. The usage of twisted mass is particularly beneficial because it provides a sharp infrared cutoff for the spectrum of the lattice operator.

We have performed simulations with a tree-level improved gauge action [19] and $N_{\mathrm{f}}=2$ Wilson twisted mass fermions [20-22]. We summarize in tab. 1 the parameters of the numerical simulations we are currently performing. To determine the LECs we compute two-point functions and we compare the Euclidean time dependence of the correlation functions with the time dependence predicted by $\chi$ PT.

In fig. 1 we show the numerical results, for the ensemble $F_{2}$, for the charged pseudoscalar density two-point function and the fit results obtained using the NLO formula in the continuum [23, 24]

$C_{\mathrm{P}}\left(x_{0}\right)=\frac{\Sigma_{\text {eff }}^{2}}{3}\left\{\frac{X_{2}\left(z_{\text {eff }}\right)}{X_{1}\left(z_{\text {eff }}\right)}+\frac{3}{F^{2}}\left(1-\frac{1}{3} \frac{X_{2}(z)}{X_{1}(z)}\right) \frac{T}{L^{3}} h_{1}\left(x_{0} / T\right)\right\}, \quad z_{\text {eff }}=2 \mu_{\mathrm{q}} \Sigma V\left(1-\frac{N_{f}^{2}-1}{N_{f} F^{2}} \bar{G}(0)\right)$,

(see ref. [8] for unexplained notations) in the fit range $10<x_{0} / a<38$. It turns out that the fit results are very stable if we change the number of data points included in the fit. We have repeated this analysis for all the simulation points of tab. 1 and the results for the chiral condensate $\Sigma$ and the pseudoscalar decay constant $F$ have been collected in fig. 2. This figure summarizes the results of simulations at 2 values of the lattice spacings, 2 different physical volumes and several values of the quark masses, thus allowing us to attempt a first understanding of the systematic errors. Together with the numerical data we also plot two vertical dashed $(\beta=3.9)$ and dotted $(\beta=4.05)$ lines, 


\begin{tabular}{|cccccc|}
\hline lattice & $\beta$ & Lattice & $a[\mathrm{fm}]$ & $a \mu_{\mathrm{q}}$ & $N_{\text {traj }}$ \\
\hline $\mathrm{I}_{1}$ & 3.9 & $16^{3} \times 32$ & 0.079 & 0.0005 & 2500 \\
$\mathrm{I}_{2}$ & 3.9 & $16^{3} \times 32$ & 0.079 & 0.00075 & 3500 \\
$\mathrm{I}_{3}$ & 3.9 & $16^{3} \times 32$ & 0.079 & 0.001 & 3135 \\
$\mathrm{~F}_{1}$ & 4.05 & $24^{3} \times 48$ & 0.063 & 0.00039 & 1755 \\
$\mathrm{~F}_{2}$ & 4.05 & $24^{3} \times 48$ & 0.063 & 0.00078 & 2316 \\
$\mathrm{~F}_{3}$ & 4.05 & $20^{3} \times 40$ & 0.063 & 0.00039 & 2500 \\
\hline
\end{tabular}

\begin{tabular}{|c|c|c|}
\hline Group & $r_{0} \Sigma^{1 / 3}$ & $r_{0} F$ \\
\hline This work & $0.595(12)$ & $0.224(12)$ \\
ETMC [25] & $0.576(23)$ & $0.183(6)$ \\
HHS [26] & $0.617(15)$ & $0.224(10)$ \\
JLQCD [27] & $0.596(10)$ & $0.217(14)$ \\
JLQCD [28] & $0.624(17)(27)$ & - \\
\hline
\end{tabular}

Table 1: Simulation parameters of the runs performed Table 2: Table comparing the results presented in where $\beta=6 / g_{0}^{2}$, the lattice spacing $a$ is determined in [25], $\mu_{\mathrm{q}}$ is the twisted mass parameter and $N_{\text {traj }}$ is the number of trajectories (with unity trajectory length) excluding the thermalization process. this proceedings with recent results obtained in the $\varepsilon$ regime using $N_{\mathrm{f}}=2$ overlap fermions and clover-type fermions. The result obtained in the $p$-regime with $N_{\mathrm{f}}=2$ Wilson twisted mass fermions by ETMC is also added for comparison.

for the two different lattice spacings, indicating the value of $\mu_{\mathrm{q}} \Sigma V$ where we enter the so called Aoki regime (see sect. 2). This value depends on the value of an unknown low energy constants, called $c_{2} \propto-W^{\prime}$ which parametrizes the $\mathrm{O}\left(a^{2}\right)$ effects. The two lines are the bound for $\mu_{\mathrm{q}} \Sigma V$ for two different lattice spacings given the indicative, but plausible, value $\left|c_{2}\right|=(400 \mathrm{MeV})^{4}$. The figure can be interpreted in the following way. The two different lattice spacings and same physical volumes (filled simbols) agree with themselves if we exclude the most chiral point at $\beta=3.9$ which is beyond the dashed line we have drawn. This might indicate that cutoff effects might be small if we keep the value of $\mu_{\mathrm{q}} \Sigma V$ larger than the $\mathrm{O}\left(a^{2} W^{\prime} V\right)$. The second effect is visible when we change the physical volume. There seems to be a discrepancy between the two volume simulated both for the chiral condensate and the decay constant. This discrepancy might indicate the inadequacy of a NLO fit when the volume is not large enough. On the other side there is a good consistency in the LECs if we change the value of the mass at the largest volume available for both lattice spacings (excluding the most chiral point already dicussed). If we take the finest lattice spacing and the largest volume as our best estimate of the LECs we obtain the preliminary results

$$
r_{0} \Sigma^{1 / 3}=0.595(12), \quad r_{0} F=0.224(12)
$$

In tab. 2 we compare our determination for the LECs with other determinations obtained in the $\varepsilon$-regime [26-28] and with the determination obtained by ETMC in the $p$-regime. We observe a very good agreement with all the determinations for the chiral condensate. For the pseudoscalar decay constant we observe a good agreement among all the determinations in the $\varepsilon$-regime, while there is some tension with the ETMC determination in the $p$-regime [25] (see ref. [29] for a review of ETMC results).

\section{Conclusions}

Probing the $\varepsilon$-regime with Wilson-type fermions is possible. We have now an analytical tool which allows us to study the combined lattice spacing, volume and mass dependence of correlation functions in the $\varepsilon$-regime, and we have computed two-point functions at NLO. We have performed several simulations in the $\varepsilon$-regime with Wilson twisted mass, using a PHMC algorithm combined with exact reweighting. This allows in principle the determination of the LECs $\Sigma$ and $F$ without contaminations from chiral logs. Systematic uncertainties can now be addressed because of the 


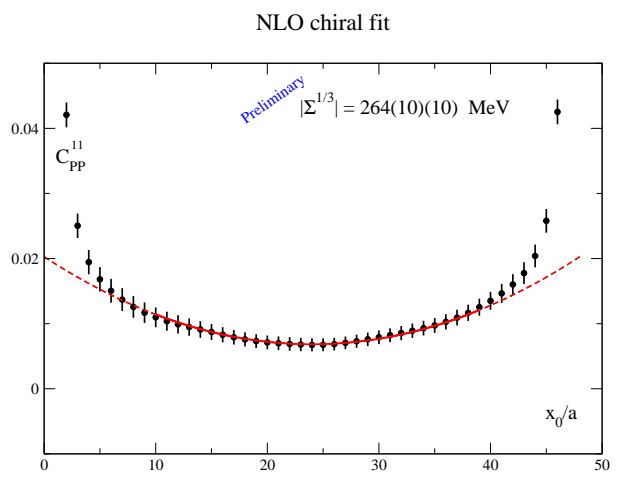

Figure 1: Euclidean time dependence of the pseudoscalar density two-point function and the fit results obtained using the NLO formula in the continuum. The fit curve becomes dashed outside the fit range.
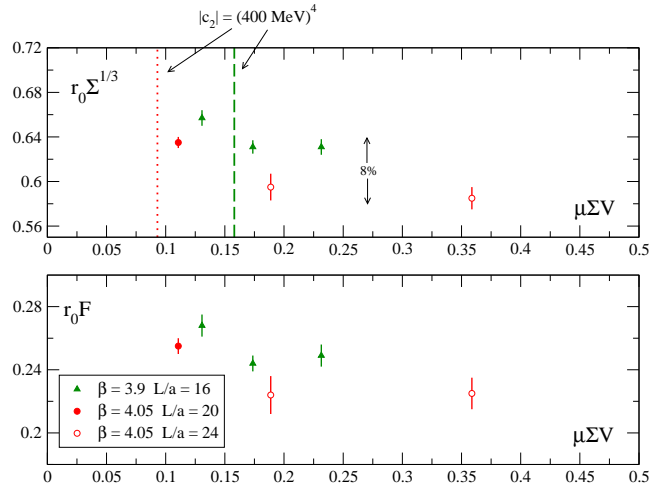

Figure 2: Plot of $r_{0}|\Sigma|^{1 / 3}$ and $r_{0} F$ for all the simulations point we have at the moment as a function of $\mu_{\mathrm{q}} \Sigma V$.

rather large set of simulations we have performed, indicating an $8 \%$ of systematic errors coming from a too small volume and a $4-10 \%$ of systematic errors coming from the finite cutoff. To ensure a reliable understanding of the systematic uncertainties and thus remove them we will enlarge the set of simulation points. We will also try to enlarge the set of physical quantities to compute to fully take advantage of the expensive dynamical simulations perfomed so far. From the analytical side we are curently extending the NLO computation performed with Wilson fermions to Wilson twisted mass [15]. We see no reason why this computation could not be done with staggered fermions, and moreover it could be a good corner of the parameter space where to test the rooting approach.

\section{Acknowledgments}

We thank the organizers of "Lattice 2009" for the very interesting conference realized in Beijing. We also aknoweledge computer time made available by CNRS on the BlueGene system at IDRIS and CCIN2P3 in Lyon, by the NW-Grid in UK and by University of Liverpool. A.S. thanks the Spanish Consolider-Ingenio 2010 Programme CPAN (CSD 2007-00042) and Comunidad Autónoma de Madrid, CAM under grant HEPHACOS P-ESP-00346 for funding.

\section{References}

[1] J. Gasser and H. Leutwyler, Chiral perturbation theory to one loop, Ann. Phys. 158 (1984) 142.

[2] J. Gasser and H. Leutwyler, Thermodynamics of Chiral Symmetry, Phys. Lett. B188 (1987) 477.

[3] P. H. Ginsparg and K. G. Wilson, A remnant of chiral symmetry on the lattice, Phys. Rev. $\mathbf{D 2 5}$ (1982) 2649.

[4] S. Hashimoto, Physics results from dynamical overlap fermion simulations, 0811.1257.

[5] P. H. Damgaard, P. Hernandez, K. Jansen, M. Laine and L. Lellouch, Finite-size scaling of vector and axial current correlators, Nucl. Phys. B656 (2003) 226-238 [hep-lat/0211020].

[6] K. Jansen, A. Nube, A. Shindler, C. Urbach and U. Wenger, Exploring the epsilon regime with twisted mass fermions, PoS LAT2007 (2007) 084 [0711 . 1871].

[7] K. Jansen, A. Nube and A. Shindler, Wilson twisted mass fermions in the epsilon regime, 0810.0300 . 
[8] A. Shindler, Observations on the Wilson fermions in the epsilon regime, Phys. Lett. B672 (2009) $82-88[0812.2251]$.

[9] O. Bar, S. Necco and S. Schaefer, The epsilon regime with Wilson fermions, JHEP 03 (2009) 006 [0812.2403].

[10] S. R. Sharpe and J. M. S. Wu, Twisted mass chiral perturbation theory at next-to-leading order, Phys. Rev. D71 (2005) 074501 [hep-lat/0411021].

[11] O. Bar, G. Rupak and N. Shoresh, Chiral perturbation theory at $O\left(a^{* * 2)}\right.$ for lattice QCD, Phys. Rev. D70 (2004) 034508 [hep-lat/ 0306021$].$

[12] S. Aoki, New phase structure for lattice QCD with Wilson fermions, Phys. Rev. D30 (1984) 2653.

[13] S. R. Sharpe and J. Singleton, R., Spontaneous flavor and parity breaking with Wilson fermions, Phys. Rev. D58 (1998) 074501 [hep-lat/9804028].

[14] F. Farchioni et. al., Twisted mass quarks and the phase structure of lattice QCD, Eur. Phys. J. C39 (2005) 421-433 [hep-lat/ 0406039 ].

[15] O. Bar, S. Necco and A. Shindler, The epsilon regime with twisted mass Wilson fermions, . In preparation.

[16] R. Frezzotti and K. Jansen, A polynomial hybrid monte carlo algorithm, Phys. Lett. B402 (1997) 328-334 [hep-lat/9702016].

[17] A. Hasenfratz, R. Hoffmann and S. Schaefer, Reweighting towards the chiral limit, Phys. Rev. D78 (2008) 014515 [0805.2369].

[18] M. Luscher and F. Palombi, Fluctuations and reweighting of the quark determinant on large lattices, 0810.0946.

[19] P. Weisz, Continuum limit improved lattice action for pure Yang-Mills theory. 1, Nucl. Phys. B212 (1983) 1.

[20] ALPHA Collaboration, R. Frezzotti, P. A. Grassi, S. Sint and P. Weisz, Lattice QCD with a chirally twisted mass term, JHEP 08 (2001) 058 [hep-lat/ 0101001 ].

[21] R. Frezzotti and G. C. Rossi, Chirally improving Wilson fermions. I: $O(a)$ improvement, JHEP 08 (2004) 007 [hep-lat/0306014].

[22] A. Shindler, Twisted mass lattice QCD, Phys. Rept. 461 (2008) 37-110 [0 707 . 4093 ].

[23] P. Hasenfratz and H. Leutwyler, Goldstone boson related finite size effects in field theory and critical phenomena with $O(N)$ symmetry, Nucl. Phys. B343 (1990) 241-284.

[24] F. C. Hansen, Finite size effects in spontaneously broken $S U(N)$ x $S U(N)$ theories, Nucl. Phys. $\mathbf{B 3 4 5}$ (1990) 685-708.

[25] R. Baron et. al., Light Meson Physics from Maximally Twisted Mass Lattice QCD, 0911.5061.

[26] A. Hasenfratz, R. Hoffmann and S. Schaefer, Low energy chiral constants from epsilon-regime simulations with improved Wilson fermions, Phys. Rev. D78 (2008) 054511 [0806. 4 586].

[27] JLQCD Collaboration, H. Fukaya et. al., Lattice study of meson correlators in the epsilon-regime of two-flavor QCD, Phys. Rev. D77 (2008) 074503 [0711. 4 965].

[28] H. Fukaya et. al., Two-flavor lattice QCD in the epsilon-regime and chiral Random Matrix Theory, Phys. Rev. D76 (2007) 054503 [0705. 3322].

[29] European Twisted Mass Collaboration, C. Urbach, Lattice QCD with two light Wilson quarks and maximally twisted mass, PoS LAT2007 (2007) 022 [0710 . 1517]. 